\title{
La Escuela Nueva en la Normal de Paraná: circulación transnacional de ideas y adaptaciones locales (1931-1937)
}

\section{A Escola Nova na Escola Normal do Paraná e suas adaptações locais (1931-1937)}

\section{The New School in the Normal School of Paraná and its local adaptations (1931-1937)}

\author{
Laura Graciela Rodríguez \\ Eva Mara Petitti**
}

\begin{abstract}
RESUMEN
En este artículo analizaremos las adaptaciones locales que se hicieron de dos experiencias que se enmarcaban en las ideas de la Escuela Nueva, llevadas a cabo en el nivel primario de la Normal de Paraná (provincia de Entre Ríos). Fueron implementadas por la pedagoga Celia Ortiz A. de Montoya primero, y la regente María del Carmen Rodríguez, después. En el primer apartado, presentaremos la experiencia realizada por Montoya en 1931 en la Escuela de Aplicación de la Normal de Paraná; y en el segundo, reseñaremos la implementación de una versión del Plan Dalton (1932-1936) que dirigió la regente Rodríguez y la inauguración de los Programas de Asuntos que se hizo partir de 1937. Pretendemos mostrar, en primer lugar, que las dos exhibían un amplio conocimiento de las ideas escolanovistas europeas y norteamericanas, lo que daba cuenta que estas nociones circulaban en el país hacía tiempo, especialmente a través de la prensa educativa. En segundo término, expondremos que ambas hicieron una minuciosa tarea de adaptación de dichas nociones a la realidad local, que las llevó a identificar claramente,
\end{abstract}

* Universidad Nacional de La Plata. Consejo Nacional de Investigaciones Científicas y Tecnológicas. La Plata, Buenos Aires, Argentina. Correo: lau.g.rodrig@gmail.com - https://orcid. org/0000-0001-9757-5609

** Universidad Nacional de Entre Ríos. Consejo Nacional de Investigaciones Científicas y Tecnológicas. Concepción del Uruguay, Entre Ríos, Argentina. Correo: marapetitti@gmail.com - https:// orcid.org./ https://orcid.org/0000-0001-8014-7181 
la distancia que existió entre las propuestas originales y la práctica concreta, y la necesidad de que el Estado, no solo las apoyara discursivamente, sino que lo hiciese con recursos y nuevas normativas.

Palabras clave: Escolanovismo. Normalismo. Argentina. Plan Dalton. Decroly.

\title{
RESUMO
}

Neste artigo, vamos analisar as adaptações locais que foram feitas de duas experiências que foram enquadradas nas ideias da Nova Escola, realizadas no nível primário da Escola Normal do Paraná (província de Entre Rios). Foram implementados primeiro pela pedagoga Celia Ortiz A. de Montoya, e depois pela regente María del Carmen Rodríguez. Na primeira seção, apresentaremos a experiência realizada por Montoya em 1931 na Escuela de Aplicación de la Normal de Paraná; e na segunda, reveremos a implementação de uma versão do Plan Dalton (1932-1936) dirigida pelo regente Rodríguez e a inauguração dos Programas de Assuntos, que teve lugar a partir de 1937. Pretendemos mostrar, em primeiro lugar, que ambas tinham um amplo conhecimento das ideias scholanovistas europeias e norteamericanas, o que mostrou que estas noções já circulavam no país há algum tempo, especialmente através da imprensa educativa. Em segundo lugar, mostraremos que as duas fizeram uma tarefa meticulosa de adaptar estas noções à realidade local, o que as levou a identificar claramente a distância que existia entre as propostas originais e a prática concreta, e a necessidade do Estado de não apenas apoiá-las discursivamente, mas de realizá-las com recursos e novas regulamentações.

Palavras-chave: Escola Nova. Normalismo. Argentina. Plan Dalton. Decroly.

\begin{abstract}
In this article, we will analyze the local adaptations of two experiences that were born in the ideas of the New School, carried out at the primary level of the Normal School of Paraná (province of Entre Ríos). They were implemented first by pedagogue Celia Ortiz A. de Montoya and then by the regent María del Carmen Rodríguez. In the first section, we will present the experience realize by Montoya in 1931 at the School of Application of the Normal of Paraná, and in the second, we will outline the implementation of a version of the Plan Dalton (1932-1936) directed by the regent Rodríguez and the inauguration of the Programs of Asuntos which took place from 1937. We aspire to show, firstly, that both had a broad knowledge of European and North American escolanovistas ideas, which showed that these notions had been circulating in the country for some time, especially
\end{abstract}


through the educational press. Secondly, we will show that both of them made a meticulous task of adapting these notions to the local reality, which led them to identify the distance between the original proposals and the concrete practice, and the role of the State and the necessity of increase the inversion and designed a new normativity.

Keywords: New Education. Normalism. Argentina. Plan Dalton. Decroly.

\title{
Introducción
}

\author{
"La escuela actual es sedentaria \\ y su símbolo más representativo \\ lo constituye el famoso banco escolar (...) \\ instrumento mecánico de precisión ortopédica" \\ Guillén de Rezzano (1934, p. 22)
}

En este artículo analizaremos las adaptaciones locales que se hicieron de dos experiencias que se enmarcaban en las ideas de la Escuela Nueva, llevadas a cabo en el nivel primario de la Normal de Paraná (provincia de Entre Ríos) y que fueron implementadas por la pedagoga Celia Ortiz A. de Montoya en 1931, y la regente María del Carmen Rodríguez, entre 1932 y 1937. ${ }^{1}$ Los especialistas han identificado que los primeros ensayos de la Escuela Nueva, se dieron por primera vez en Europa y Estados Unidos a fines del siglo XIX en escuelas privadas, y se fueron trasladando al sistema público desde la primera post guerra en 1918, de la mano de John Dewey, Georg Kerchensteiner, Carleton Washburne y Helen Parkhurst en Estados Unidos, María Montessori en Italia, Ovidio Decroly en Bélgica y Adolphe Ferrière en Suiza, entre muchos otros (LUZURIAGA, [1943] 1958; FILHO, 1964; CARUSO, 2013). De todos ellos, fue Ferrière quien, en 1930, enviado por el Bureau International de l'Education, estuvo en Argentina y otros países sudamericanos, con el propósito de documentar las experiencias escolanovistas. En Argentina, visitó una considerable cantidad de escuelas y dio numerosas conferencias en distintas ciudades, por lo que se convirtió en

1 Agradecemos a los colegas que han leído y comentado este artículo: Alejo Levoratti, Antonela Centanni, Asunción Iglesias, Bernardo Carrizo, Darío Velázquez, Germán Soprano, José Bustamante Vismara, Juan Cruz Giménez, Luciana Garatte, Maximiliano Camarda, Nerina Visacovsky y Pamela Reisin. 
un gran difusor de la Escuela Nueva entre los maestros y profesores de todo el país que lo fueron a escuchar. Producto de su viaje publicó La Escuela Activa en América Latina (FERRIÈRE, 1936). ${ }^{2}$ En este trabajo, antes que estudiar en profundidad estas nociones extranjeras, nos interesa observar cómo circulaban y eran apropiadas e interpretadas por los argentinos. ${ }^{3}$

¿Y cómo llegaron estas ideas a la Argentina? Maestros, profesores y académicos, se fueron enterando de la existencia de la Escuela Nueva por distintas vías: a través de la prensa pedagógica internacional como la Revista de Pedagogía de Madrid; porque habían viajado a Europa y Estados Unidos y se habían contactado con algunos de estos pedagogos o sus discípulos; leyendo las notas que aparecían en la revista $\mathrm{La} O \mathrm{Obra}$, publicación fundada en 1921 con el propósito de difundir los preceptos de esta Escuela; revisando la publicación oficial El Monitor de la Educación Común; comprando materiales específicos de editoriales como Kapelusz; o bien porque habían asistido a alguna de las conferencias organizadas durante la década de 1930, donde expusieron importantes referentes extranjeros y argentinos (GVIRTZ, 1996; GVIRTZ; BAROLO, 2020; FRECHTEL, 2020). Existe un consenso entre los estudiosos de la Escuela Nueva, acerca de que distintos normalistas, sobre todo entre 1920 y 1940, llevaron a cabo innovaciones muy relevantes, pero que en general, resultaron breves en el tiempo, tuvieron el carácter de micro experiencias, estuvieron aisladas entre sí e involucraron solo algunos grados de primaria y/o salas de Jardín de Infantes (TEDESCO, 1993; PUIGGRÓS, 1992; GVIRTZ, 1996; NARODOWSKI, 1996; CARLI, 2002). ${ }^{4}$ Se han identificado también influencias escolanovistas en algunas escuelas rurales y en ciertos libros de lectura de la década de 1930 (ASCOLANI, 2010, 2017).

En esos años, junto al avance de la Iglesia Católica en el campo de la educación y el auge de la filosofía espiritualista, es posible identificar, al interior del Ministerio de Instrucción Pública, funcionarios que impulsaron estas teorías

2 Sobre la biografía de Ferrière y su viaje, ver, entre otros, Caruso (2013) y Frechtel (2017).

3 En este artículo utilizaremos en la redacción el genérico masculino clásico para no sobrecargar la escritura -especialmente en este caso, que se trata de una escuela mixta- en el entendido que incluye siempre a varones y mujeres.

4 Según Gvirtz y Bartolo (2020) hubo experiencias de Escuela Nueva en los años de 1960 en algunas escuelas privadas de la ciudad de Buenos Aires y parte de sus principios fueron retomados en las reformas que se hicieron en los años de 1990. Por su parte, Levoratti (2021) ha mostrado que las reformas de la asignatura de Educación Física que hizo el Estado en la década de 1960, también se referenciaban en la Escuela Nueva. En la actualidad, en Argentina y en otros países, existen numerosos establecimientos de nivel inicial, primario y secundario, que siguen los postulados de la Escuela Nueva y en general, son Jardines y escuelas de carácter privado donde se abonan matrículas y cuotas muy onerosas. 
de la Escuela Nueva, en tanto interpretaban que representaban una reacción al positivismo en la enseñanza, que asociaban al "laicismo" y al "ateísmo" (PUIGGRÓS, 1992; ASCOLANI, 2010, 2017). Ciertamente, los pedagogos europeos se mostraban de acuerdo con la incorporación de la religión en sus establecimientos para "contribuir al desarrollo espiritual" de los estudiantes, debiendo impartirse de manera "no sectaria" y "tolerante" hacia otros credos (FILHO, 1964). De todos modos, los docentes que llevaron a cabo experiencias innovadoras en distintos lugares del país, tuvieron diferentes adscripciones, y los hubo católicos, pero también laicistas y socialistas, entre otras (PUIGGRÓS, 1992; ASCOLANI, 2010, 2017).

En general, los investigadores coinciden en señalar que en Argentina, los normalistas más representativos de esta corriente fueron: José Rezzano y Clotilde Guillén de Rezzano (ciudad de Buenos Aires); Olga y Leticia Cossettini, y Dolores y Bernardina Dabat (Santa Fe); Florencia Fossatti (Mendoza); Antonio Sobral y Luz Vieyra Méndez (Córdoba), Luis Iglesias (Buenos Aires); y Celia Ortiz A. de Montoya en Entre Ríos (PUIGGRÓS, 1992; GVIRTZ, 1996; GVIRTZ; BAROLO 2020; ROITENBURD, 1996; ROMÁN, 1998, 1999; CARLI, 2002; CUCUZZA, 2017; GIMÉNEZ, 2020; OLANO, 2020). ${ }^{5}$ Estos docentes proponían superar las prácticas de la "escuela tradicional", donde, entre otras cosas, el maestro era la figura central del aula, quien brindaba solo clases expositivas. Los alumnos permanecían la mayor parte del tiempo en silencio, sentados en unos bancos que les permitían realizar pocos movimientos, y solo podían preguntar cuando el docente terminaba de hablar. Al estudiante se le exigía básicamente que memorizara contenidos de tipo enciclopédico que rara vez el docente intentaba vincular con su vida cotidiana, y era evaluado por medio de pruebas estandarizadas (REZZANO, 1934).

De acuerdo a algunos analistas, de todos estos ensayos innovadores, la obra de la pedagoga Montoya expresaba una de las versiones más representativa de la Escuela Nueva (CARLI, 2002; OLANO, 2020) y con su clausura a fines de 1931, interpretaron que el escolanovismo en Paraná había llegado a su fin (PUIGGRÓS, 1992; ROMÁN, 1998, 1999; CARLI, 2002; OLANO, 2020). Sin embargo, como ya mencionamos, al ensayo de Montoya le siguió el de la regente Rodríguez, que resultó mucho menos conocido pero que se mantuvo, por

5 A este listado, Puiggrós (1992) ha agregado otras experiencias innovadoras implementadas por Hugo Calzetti, Horacio Ratier, Javier Villafañe y Delia Etcheverry; y Cucuzza (2017) ha añadido a la maestra Anunciada Masitelli. Disponemos de un abundante número de trabajos sobre las hermanas Cossettini en particular y acerca de otras experiencias en Santa Fe. Ver, entre otros, Ziperovich (1992); Caldo y Fernández (2010); Pellegrini y Mosso (2016); Pellegrini (2016); Dip (2017); Welti y Serra (2018). Sobre la experiencia de Iglesias, ver Padawer (2017). 
lo menos, hasta fines de la década. La experiencia de Montoya también fue muy elogiada por el suizo Ferrière, que conoció personalmente a la pedagoga cuando ella estuvo en Europa entre diciembre de 1925 y abril de 1926, visitando distintos establecimientos de España, Italia y Suiza (ROMÁN, 2006). Lo realizado por Montoya, afirmaba, había hecho de Paraná "una de las ciudades pedagógicas más ricas del continente" (FERRIÈRE, 1936, p. 95). Su obra, seguía, tenía "un valor verdaderamente universitario, es decir, científico" que demostraba que se trataba de "uno de los más bellos ensayos de Escuela Activa en América Latina", tanto más meritorio cuanto servía "de campo de acción y difusión a centenares de futuros maestros y maestras que se diseminarán por el ámbito de la inmensa Argentina" (FERRIÈRE, 1936, p. 96). Asimismo, recomendaba enfáticamente los escritos de Clotilde Guillén de Rezzano, que ya había publicado en la Revista de Pedagogía de Madrid y el libro Hacia la Escuela Activa, del año 1934, título que retomaba la denominación que utilizaba el suizo, que hablaba de Escuela Nueva o Escuela Activa.

Algunas de las características que debían tener las Escuelas Nuevas, explicaba el suizo, eran: "trabajo manual de ebanistería y cultivo de la tierra, régimen de autonomía escolar, coeducación de sexos sin limitación, favor concedido a la iniciativa y a los trabajos libres, juegos y deportes, viajes instructivos, fomento de aptitudes dominantes con el fin de orientación profesional subsiguiente, educación científica experimental e intuitiva, educación moral teórica y práctica" (FERRIÈRE, 1936, p. 92). A este listado incompleto, debía añadírsele la aplicación de test psicológicos individuales para reconocer a los niños con problemas de aprendizaje. Finalmente, Ferrière se expresaba optimista sobre la situación de Argentina, porque José Rezzano había asumido como vocal del Consejo Nacional de Educación y había sido uno de los inspiradores de los Programas de Asuntos que se aplicaron en algunas escuelas primarias de la ciudad de Buenos Aires. Este primer ensayo se extendió en 1937 a todas las escuelas primarias nacionales y resultó un intento de ciertos funcionarios públicamente religiosos, de institucionalizar y hacer masivos los principios de una Escuela Nueva "argentinizada" para el sistema público oficial. En esta empresa fueron apoyados de manera entusiasta por los redactores de La Obra (PUIGGRÓS, 1992; GVIRTZ, 1996; GVIRTZ; BAROLO, 2020; FRECHTEL, 2020).

Ahora bien, este artículo contiene dos apartados, en el primero presentaremos la experiencia realizada por Montoya en 1931 en la Escuela de Aplicación de la Normal de Paraná; y en el segundo, la continuidad que le dio a este ensayo la regente, María del Carmen Rodríguez, con la implementación de una versión del Plan Dalton (1932-1936) y la inauguración de los Programas 
de Asuntos que hizo a partir de $1937 .{ }^{6}$ Para ello, nos valdremos de un trabajo publicado de Montoya y de los informes de la Regencia de la Escuela de Aplicación, los cuales nos permiten presentar las ideas programáticas del escolanovismo según la interpretación de las docentes, pero sobre todo, cómo se intentaron plasmar en la práctica, de acuerdo a sus protagonistas. Pretendemos mostrar, en primer lugar, y en base a las investigaciones precedentes, que esta experiencia realizada en Paraná se destacó dentro del normalismo, porque resultó una de las pocas que tuvo cierta estabilidad en el tiempo - más de seis años - y abarcó todos los grados de la primaria - de primero a sexto-. En segundo término, señalaremos que las dos exhibían un amplio conocimiento de las ideas escolanovistas europeas y norteamericanas, lo que daba cuenta que estas nociones circulaban en el país hacía tiempo, especialmente a través de la prensa educativa. En tercer lugar, indicaremos que ambas hicieron una minuciosa tarea de adaptación de dichas nociones a la realidad local, que las llevó a identificar claramente, la distancia que existió entre las propuestas originales y la práctica concreta, y la necesidad de que el Estado, no solo las apoyara discursivamente, sino que lo hiciese con recursos y medidas específicas. Expondremos que, del lado de los avances, las docentes lograron flexibilizar algunos horarios y disposiciones, conseguir y renovar materiales, e innovar en algunas pautas organizacionales, mientras que, del lado de los impedimentos, estos estuvieron vinculados a la infraestructura de la Escuela de Aplicación, a la escasez de materiales, la actitud de las familias, las normativas oficiales y a la poca articulación con los otros niveles de la Escuela Normal.

\section{"Nuestro ensayo de Escuela Nueva" (1931)}

A comienzos del siglo $\mathrm{XX}$ se intentaron realizar varios cambios institucionales en la Normal de Paraná, que finalmente no prosperaron. En la década de 1920 se puso al establecimiento bajo la órbita de la recientemente creada Facultad de Ciencias Económicas y Educacionales (1920-1931) dependiente de la Universidad Nacional del Litoral (MONTOYA, 1967;

6 La Escuela Normal de Paraná fue la primera institución normalista de nivel nacional creada en el país, a instancias del presidente Domingo F. Sarmiento. Fue inaugurada en agosto de 1871. Sobre la historia de esta Normal, ver entre otros, Figueroa (1934); Carli (1993); Bosch (1993); Pérez Campos (2016); De Miguel et al. (2017). Una historia de largo plazo (1871-1969) está en Rodríguez y Petitti (2021). 
ROMÁN, 1998; KUMMER, 2010; OSSANNA et al., 2012). En ese contexto, Montoya, que era profesora egresada de la Normal de Paraná y doctora en educación por la Universidad Nacional de La Plata, fue designada directora del Instituto de Pedagogía en 1930, que se había fundado el año anterior dentro de la Facultad. ${ }^{7}$ Siendo directora, propuso la realización del ensayo escolanovista, que se implementó durante todo el ciclo lectivo de 1931. El problema fue que, en el transcurso de ese año y por distintas razones, la Facultad y el Instituto fueron cerrados, y la Normal salió de la dependencia universitaria (IBARLUCÍA, 2018).

A poco de comenzar el ciclo lectivo del año 1932, el director de la Escuela Normal Superior, Roberto Escobar, decidió clausurar la experiencia de Montoya y que fuese la regente Rodríguez quien la continuara e inaugurara una nueva. Sobre este episodio, Montoya contó en una entrevista que Escobar la había maltratado y le había cerrado la posibilidad de continuar con el ensayo "porque interfería con el que realizaba la Dirección de la Escuela" (cit. en ROMÁN, 2006, p. 11). En uno de sus libros añadía, que, luego de un año, le "llegó la prohibición de continuarlo progresivamente en los demás grados como proponía el proyecto aprobado", permitiendo en cambio "continuar con la aplicación del Test Vermeylen" para medir las capacidades de los niños (MONTOYA, 1967). ${ }^{8}$

Montoya dio a conocer el detalle de la experiencia desarrollada en su artículo publicado en 1933 en el Boletín de Educación de la provincia de Santa Fe, titulado: "Nuestro ensayo de Educación Nueva". ${ }^{9}$ Allí relataba que, siendo directora del Instituto de Pedagogía, solicitó al Consejo Directivo permiso para iniciar en la Escuela de Aplicación de la Normal un ensayo de "educación integral activa". Creyó oportuno, explicaba, solicitar dicho permiso para salir del mundo de la teoría y pasar a la práctica concreta, que pusiera como piedra angular "la auto elaboración y la auto expresión" del niño (MONTOYA, 1933, p. 51). El propósito principal era organizar las actividades escolares llevando un

7 Montoya egresó en 1915 de la Normal de Paraná como maestra y en 1918 se graduó como profesora de Pedagogía y Filosofía. Tras cursar estudios en la Universidad Nacional de La Plata en 1921 obtuvo el Doctorado en Ciencias de la Educación, siendo la primera mujer con ese título. En 1922, ya en Paraná, ingresó con el cargo de docente interna en las cátedras de Historia de la Educación, Didáctica General y Práctica de la Enseñanza en la Facultad de Ciencias Económicas y Educacionales. En 1929 fue nombrada titular mediante concurso de la cátedra de Historia de la Educación. En 1931 se hizo cargo de las cátedras de Didáctica General, Ética y Seminario, siguiendo su actividad docente en el Instituto Nacional del Profesorado Secundario. Montoya, a diferencia de Rodríguez, contaba con una abundante obra pedagógica y era una referente de su disciplina en el país.

8 De acuerdo a Rodríguez Mata (1934), el test del médico belga Vermeylen era de tipo cualitativo y se aplicaba sobre todo para la exploración de los niños en esa época descriptos como "deficientes" y "anormales".

9 Agradecemos a Juan Cruz Giménez habernos facilitado el artículo. 
“control experimental del desarrollo corporal, intelectual, moral, social, estético y expresivo de los educandos, para apreciar en todo momento el progreso realizado, por medio de la aplicación sistemática de tests psicológicos" (MONTOYA, 1933, p. 63). Como ella misma lo definía, su ensayo era una mezcla de las teorías de Decroly con las de Montessori principalmente, donde se utilizaba material especialmente diseñado por ella.

Acerca del desarrollo del proyecto, contaba que se aplicó en primer grado inferior y contemplaba: gimnasia natural, juegos libres y dirigidos, vida al aire libre, gimnasia metódica y rítmica, audiciones musicales y artísticas, canto coral, danza nacional, plástica, juguetes cómicos, decoración del aula, aquariums y terrariums. Se pretendía organizar la clase en grupos, estimulando el autogobierno y la cooperación y para ello, el Instituto había adquirido con fondos propios unas mesas pequeñas azul claro con sus sillas y armarios bajos - para permitir que los niños guardasen sus materiales -, atriles, cajas de madera y un fichero con 36 casillas.

La pedagoga había solicitado trabajar solo con 18 niños, pero las autoridades se lo impidieron y debió realizar la experiencia con los 70 que estaban inscriptos, repartidos en dos turnos, debiendo además, cumplir con el programa oficial. Montoya reconocía que el trabajo con 35 niños hizo que la tarea se complicara y no pudiera realizarse en las condiciones que establecían algunos de los referentes de la Escuela Nueva, debido sobre todo a la escasez del espacio y ciertas dificultades para mantener el orden. Sin embargo, rescataba que por lo menos habían podido sustituir "los odiosos pupitres" que impedían la colaboración y el libre movimiento y lograron que alrededor de cada mesa se reuniesen niños en grupos de seis.

En cuanto a los horarios, tuvieron que respetar las horas de entradas y salidas del establecimiento y seguir los programas oficiales, aunque lograron cierta libertad para hacer los ajustes necesarios y alcanzaron su principal objetivo: que solo una hora diaria fuese destinada para labores intelectuales - técnicas, lográndose una integralidad expresiva bastante aceptable (Cuadro 1). A veces, se sustituía todo un día con una excursión y su consiguiente expresión gráfica. Para conseguir un ambiente debidamente oxigenado, hicieron frecuentes salidas a la plaza vecina, donde en la primera hora los niños dibujaban, leían o participaban de actividades lúdicas. 
CUADRO 1 - HORARIO DE LA "ESCUELA INTEGRAL ACTIVA"

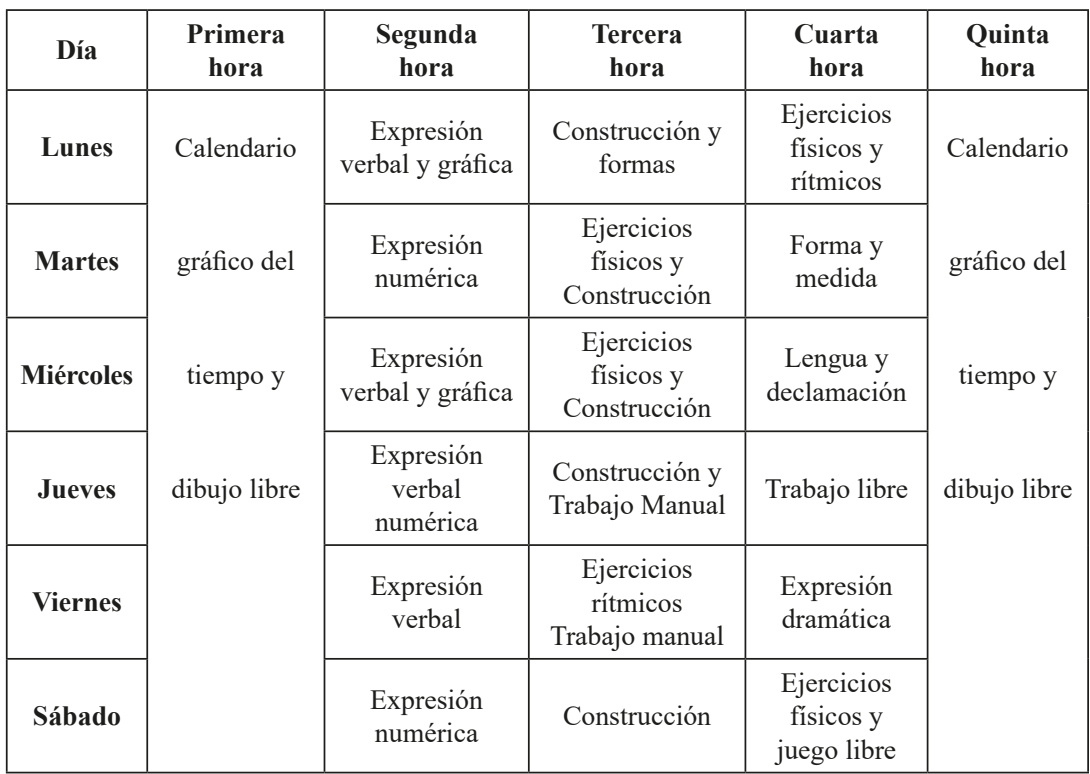

FUENTE: elaboración propia en base a MONTOYA (1933, p. 69).

Trabajaron también con una mesa de arena, donde los niños construyeron "ora un conejo, un pollito que observaban y cuidaban", trazaron "el río, levantado un túnel, simulado un desierto y una huerta" (MONTOYA, 1933, p. 66). Todo el "material auto educador", seguía Montoya, decidió diseñarlo y construirlo junto con las maestras, porque no existía tal cual lo necesitaba. Todo fue "pacientemente preparado para el ensayo y donado al Instituto" por las profesoras Fayó, Pons, Segovia, Monzón y Rubinstein.

Otra limitación que debieron sufrir, se quejaba Montoya, fue la ausencia absoluta de tierra para las experiencias botánicas infantiles, lo que les impidió hacer observaciones individuales sobre cuatro plantas por año, tal como tenían previsto. Tuvieron que reemplazar esta carencia por la siembra en macetas o poner semillas y papas en vasos. Asimismo, faltaron talleres para realizar trabajos de plástica, de madera y cartón, por lo que fue habilitada la galería contigua al grado y tuvieron a disposición unos serruchos, martillos y tablas adquiridos por la dirección. En el futuro, decía, era necesario dotar a los grados posteriores de una imprenta, un telar, un torno de cerámica y una pequeña cocina, antesala natural del laboratorio de Física y Química. 
Montoya comentaba a continuación, las excursiones que hicieron con los niños a los puestos de mercado, donde las maestras los dejaban actuar según sus intereses reales. El "centro de actividades", en la experiencia descripta por la pedagoga, fue el mercado central y las diferentes estaciones del año. Aclaraba que este no era simplemente un "centro de interés" como los había planteado el belga Decroly. En su ensayo se trataba de unir los valores morales, sociales y dinámicos de los "proyectos", con los valores expresivos ideados por Decroly, acentuando la atmósfera familiar con la estética de la escuela italiana de Montessori y la rítmica musical del ginebrino Dalcroze (MONTOYA, 1933, p. 73). ${ }^{10}$ De esta manera, en marzo, les enseñaron a los alumnos los canastos llenos de uvas frescas, en junio las verduras y en julio las carnes rojas. El día que fueron a ver las carnes, rememoraba, los niños se detuvieron en el puesto de pescado, parada no prevista, pero la educadora y sus colaboradoras decidieron cambiar el propósito de la actividad, respetando el interés de los niños, cautivados por los pacúes, dorados, pejerreyes, mojarritas, bogas, pulpos y almejas, y terminaron haciendo todas las tareas alrededor de los peces. Se preguntaba por qué condenar a niño a trabajar a medias dentro del aula con temas que no entraban en el centro de su interés, como hacía la escuela tradicional. Otras excursiones que habían realizado fueron a un vivero, a la casa de un avicultor y a una quinta suburbana.

En las clases, seguía, se introdujo el dibujo libre, sin enseñanza de técnicas ni imitación de modelos hechos (copia), en tanto se producía una "inhibición psicomotora" cuando una persona mayor le indicaba a un niño cómo se hacía un dibujo (MONTOYA, 1933, p. 77). Asimismo, la expresión manual también fue libre. Cuando volvieron del mercado y decidieron que iban a decorar el aula con uvas recordando a la estación del otoño, las maestras dejaron en libertad a los niños para que midieran las paredes del aula, decidieran cuántos racimos había que dibujar y los hicieran según sus pareceres, inventado técnicas nuevas y permitiéndoles innovar en tamaños y colores. Esta expresión plástica del otoño fue acompañada de cantos, poesías colectivas, bailes alusivos y gimnasia rítmica. A la llegada del invierno, decidieron decorar el aula con naranjas y mandarinas, y no sacar las uvas.

En relación a cómo enseñar la lecto escritura, explicaba que la única $y$ verdadera forma de cultivar el lenguaje era plantear juegos de lenguaje espontáneos y comentarios jocosos que se enriquecían con el cuento, la poesía, la expresión musical y la dramatización. Respondiendo a esa necesidad psicológica,

10 Un análisis sobre los otros referentes que influyeron en el pensamiento de Montoya, está en Olano (2020). 
la iniciación a la lectura fue "global". ${ }^{11}$ La escuela tradicional, criticaba Montoya, iniciaba este proceso con palabras elegidas por el maestro, yendo de las más simples (nene, oso) a descomponer la palabra para luego combinarla para formar otras. Este método, creía, destruía el hablar del niño y lo iniciaba en el arte de hacer frases sin decir lo que deseaba. La "lectura global" permitía usar formas de lenguaje usuales como "Dame uvas". El proceso de lectura- escritura, aclaraba, nunca fue hecho como en la escuela tradicional, con un cuaderno de caligrafía, sino que estaba unido al centro de actividades habituales, sin romper la unidad del pensar, el hablar y el escribir, combinando letras y números cuando era necesario. Con ello habían logrado que el alumno leyese y escribiese la frase que había necesitado pronunciar, por ejemplo, cuántas uvas había en un racimo, los kilos que se compraron del mercado y las monedas que se utilizaron para pagarlas. La pedagoga relataba que iniciaban a los alumnos en la lectura y en la escritura a partir de la construcción de frases en tiras de papel, seguidos de ejercicios de dictado, hasta llegar al período de lectura de cuentos ilustrados con leyendas cortas y largas, extraídas de las revistas infantiles que los niños frecuentaban. Las frases de las revistas eran pegadas en las hojas de sus cuadernos y resultaban su primer libro de lectura verdaderamente infantil, es decir, más infantil que los libros que circulaban. Posteriormente, las reproducían en la arena, el cartón y/o la madera y construían carros, jardineras, canastos, jaulas, gallinas, gallos, chanchos, patos, pavos, peces, huerta, verduras, frutas y herramientas.

Por otra parte, varios representantes de la Escuela Nueva europea y norteamericana, creían muy importante que se aplicaran distintos tipos de tests individuales para conocer las características de cada niño, identificar a los "retrasados" y seguir la evolución de los aprendizajes de los llamados "normales". Si bien Montoya aplicó el test Vermeylen destinado a clasificar a los niños, consideraba que este test era una medida demasiado relativa para determinar una clasificación definitiva, ya que muchos evolucionaban rápidamente pasada la crisis de crecimiento y otros, a la inversa. Los niños que se habían identificado como "retardados", decía la pedagoga, debían estar en aulas con mucho menos alumnos, aunque la educación integral que ella proponía, no necesariamente debía "eliminar" a quienes denominaba "no dotados". En este sentido, consideraba que faltaban escuelas para niños débiles, cursos de aceleración para aquellos que tenían una escolaridad deficiente o lentitud de desarrollo mental, y colonias de vacaciones.

11 Según algunos analistas, esta forma se contrapuso al "método torrista" de enseñanza, ver Román (2011). La manera en que la Escuela Nueva propuso renovar los métodos de enseñanza de la lectura, está en Braslavsky [1962] (2014); y Gvirtz (1998), entre otros. 
Finalizaba afirmando que la escuela tradicional, en aras "del intelectualismo abstracto, pedagocéntrico y dominador" introducía al pequeño a "un mundo sin sentido" (MONTOYA, 1933, p. 88). Ella y las maestras, explicaba, compelidas por los programas oficiales, habían tenido que avanzar igual, pero lo cierto era que los niños de Paraná, ese año, salieron muy distintos de otros escolares, en tanto, a partir de esta experiencia, habían crecido "en espíritu, en nobleza" y "en amor" (MONTOYA, 1933, p. 93).

\section{EI Plan Dalton (1932-1936) y los Programas de Asuntos (1937)}

La segunda experiencia de Escuela Nueva fue dirigida por la nueva regente (desde 1931) de la escuela primaria, María del Carmen Rodríguez y en los hechos, resultó una continuidad del ensayo de Montoya que se había llevado a cabo en el primer grado inferior. ${ }^{12}$ Cabe añadir que para esa misma época, la directora del Jardín de Infantes de la Normal de Paraná, Hortensia Wybert, junto con las maestras Sara Haedo y Virginia Arengo, habían iniciado unos años antes, en 1928, una adaptación de los "centros de interés", junto con los principios de Froebel y Montessori, intentando vincular la tarea con la que se estaba llevando a cabo en primer grado de la Escuela de Aplicación. Habían planteado el desarrollo de "centros" como "Las estaciones", "Los vestidos" y "Los alimentos", entre otros.

Según explicaba Rodríguez en su informe, en 1932, se siguió la experiencia en primer grado superior, y en 1933, se había continuado en primero inferior, segundo y tercer grados, donde adaptaron y desarrollaron los siguientes "centros de interés" de Decroly: "La ciudad de Paraná" (primero y segundo grados) y "El Río Paraná" (tercer grado) (RODRÍGUEZ, 1934). Comunicaba que se estaba enseñando, de primero a cuarto grado de la clase de música, la rítmica del sistema Dalcroze que "tan excelentes resultados ha dado desde el año 1931", cuando lo iniciara Montoya.

La regente Rodríguez informaba, además, que en quinto grado se comenzó a aplicar una versión del Plan Dalton. Ella lo había conocido a partir de la difusión que le daba la revista La Obra y los facsímiles publicados por la editorial Kapelusz. Este sistema había sido creado por Helen Parkurst en la localidad

12 Rodríguez era egresada de la Normal de Paraná y por lo menos desde 1920, ejercía como docente en la institución. La abreviatura s/p significa sin número de página. 
norteamericana de Dalton, y estaba diseñado para los grados superiores de la primaria. Clotilde Guillén de Rezzano (1934), en el libro que había ponderado enfáticamente el suizo Ferrière, explicaba en detalle de qué se trataba el método y brindaba algunos ejemplos prácticos que se podían seguir. La profesora Rezzano decía que la libertad y la responsabilidad eran los ejes del sistema, ya que el niño debía organizar un plan de trabajo de acuerdo con sus preferencias, facilidades y dificultades. En el marco de lo previsto por el Plan, el estudiante recibía por escrito las "asignaciones" que detallaban las actividades a realizar: observaciones, lecturas, redacciones, dibujos, modelados y construcciones; se le indicaban las conferencias que debía escuchar y las visitas a diversos lugares que tenía que realizar. Dicha "asignación" funcionaba al mismo tiempo como un "contrato" donde el estudiante se comprometía a realizar las tareas. De toda su labor individual y grupal, el estudiante debía presentar trabajos escritos a su maestro, quien le hacía las observaciones y las críticas correspondientes. Si el trabajo era satisfactorio, podía seguir "avanzando" y recibía una o dos unidades más, según lo establecía la "asignación". Cada materia debía tener su aula y cada salón su docente especializado, que dirigía simultáneamente el trabajo de niños que pertenecían a secciones diferentes. El maestro debía evitar dar clases expositivas y simultáneas como en la escuela tradicional, tenía que concentrarse en revisar las unidades de trabajo terminadas por cada alumno, contestar las preguntas, dilucidar los puntos obscuros, orientar las discusiones y disertar ocasionalmente sobre un tema que interesara a un grupo. Su actitud, como en el caso de la maestra montessorina, tenía que ser expectante, de guía y no de maestro que hablaba solo frente a una clase totalmente pasiva (REZZANO, 1934).

En Argentina, distintas maestras estaban implementando en esos años versiones del Plan Dalton combinadas con otros referentes teóricos, en escuelas de las provincias de Santa Fe y Buenos Aires, por ejemplo (ZIPEROVICH, 1992; CUCUZZA, 2017). Por su parte, editoriales como Kapelusz estaban vendiendo propuestas didácticas para cuarto, quinto y sexto grado, llamadas Daltonizando la escuela. Al respecto, la maestra Ziperovich de Santa Fe, admitía que una de las dificultades mayores que tenían para implementar esta innovación, era que el equipamiento para llevarlo a cabo demandaba una infraestructura especial y muchos recursos económicos, porque se necesitaban aulas para cada asignatura, laboratorios, libros, mapas, aparatos y láminas para cada estudiante. Además, si la maestra no podía comprar las "asignaciones", debía elaborar ella misma los cartones para cada alumno o grupo (ZIPEROVICH, 1992).

Ahora bien, en la Normal de Paraná, la regente relataba en su informe anual que se habían organizado en quinto grado tres Laboratorios con materias "daltonizadas": el Laboratorio de Matemática (Aritmética, Geometría, Física y Dibujo); el Laboratorio de Letras (Lenguaje, Historia, Geografía, Instrucción 
Cívica) y el Laboratorio de Áreas Naturales, (Mineralogía, Botánica, Zoología y Cuerpo Humano) (RODRÍGUEZ, 1934). Debido a la imposibilidad de contar con un aula para cada materia, se hicieron estas asociaciones y se trabajó sobre todo en grupo. Afirmaba la regente, que la enseñanza, sin ceñirse estrictamente a las formas del trabajo establecidas en el método Decroly o en el Plan Dalton, ha tomado de dichos sistemas lo adaptable a las condiciones y propósitos de la enseñanza normal y oficial y por ello, el plan de trabajo se cumplía forzosamente en forma incompleta y limitada (RODRÍGUEZ, 1934). Es decir, desde primer grado hasta sexto, estos ensayos se realizaban dentro de las limitaciones que derivaban del horario, los programas, el régimen de promociones y las condiciones del local.

El tiempo escolar, de acuerdo al Plan, se dividía en "libre" (16 horas y media) y "obligatorio" (10 horas y media). El desarrollo completo del programa de cada materia estaba organizado en "asignaciones". En ellas, la semana o período de trabajo estaba formado por seis unidades divididas de acuerdo al esfuerzo que la tarea asignada exigía. Para controlar el trabajo contaban con una "tarjeta del alumno" que permitía consignar lo realizado cada bimestre. La regente exponía que cada sección contaba con su biblioteca en el aula para consultar los libros que se necesitaran, sin embargo, había un número muy limitado de ejemplares, lo que entorpeció en ciertos momentos el trabajo, alterando los planes de labor diario de los alumnos y el ambiente de tranquilidad en que debía realizarse la actividad requerida. Afortunadamente, seguía, el Laboratorio de Matemática poseía el material necesario para la experimentación en Física; en el de Naturales los alumnos tenían a disposición los ejemplares del Museo; y las salas de Letras y Matemática, disponían de suficiente material para las proyecciones.

Rodríguez admitía que al principio, igual que ocurrió en otras escuelas, este ensayo no fue bien recibido por las familias: "Al entusiasmo de los profesores por realizar esta obra de indudable beneficios, que les imponía un esfuerzo sincero y siempre renovado, se ha opuesto con excesiva frecuencia la incomprensión o la indiferencia de los padres" (RODRÍGUEZ, 1934). Para la mayoría de los padres, señalaba la regente, el problema de la educación de sus hijos se relacionaba con la cantidad de conocimientos adquiridos, sin preocuparse por la forma de adquisición y la disciplina intelectual y moral que cada forma de trabajo implicaba. Por lo tanto, añadía, la colaboración del hogar era con frecuencia negativa por falta de confianza en la obra del maestro. Entre los "malos hábitos" de algunos estudiantes, destacaba que no se animaban a manifestar sus dificultades al profesor, se copiaban de los trabajos de sus compañeros y hacían las tareas en sus casas, en vez de realizarlas en el aula. 
Para revertir esta situación, Rodríguez convocó a una reunión de padres para explicarles las características y los propósitos del plan, manifestando que la colaboración que les reclamaba del hogar, consistía en no permitir que se hicieran trabajos fuera de la escuela. Como resultado de encuentro, indicaba, algunos hogares respondieron con amplio espíritu de comprensión a este llamado y los alumnos cambiaron de actitud.

La regente admitía que parte del malentendido se originó porque las "asignaciones" propuestas al principio, aunque muy meditadas entre los maestros y elaboradas por ellos, exigían un trabajo superior al que el alumno podía realizar en el tiempo que la distribución horaria asignaba para cada materia. Ello explicaba el nerviosismo de los estudiantes y sus familias, y el hecho de que solo un treinta por ciento de ellos hubiera podido realizar la ejercitación correspondiente al bimestre. Sin embargo, hechos los ajustes necesarios, afirmaba que los alumnos habían aprendido a estudiar de otra manera.

En cuanto a la disciplina, la libertad de movimientos en los Laboratorios y la responsabilidad del autogobierno, Rodríguez mencionaba que no habían tenido problemas serios. Por el contrario, aseguraba que era posible alcanzar por este camino, una disciplina real, ajena a la imposición constante y a la enseñanza permanente del castigo. Señalaba que al terminar el ciclo lectivo, las opiniones de los alumnos eran en su mayoría favorables al sistema y destacaba, como factor decisivo del éxito obtenido, el entusiasmo y la laboriosidad del personal que participó en el ensayo. El director de la Normal, Gabriel Echenique, había dicho que la experiencia que había llevado a cabo revelaba un trabajo inteligente y sincero, y gracias a ello, la regente fue autorizada a continuar el ensayo en sexto grado.

A comienzos de 1936 destacaba que, luego de tres años de experimentación en esta forma de trabajo que proponía el Plan Dalton (1933, 1934 y 1935), numerosos padres veían con satisfacción el trabajo de sus hijos y acordaban plenamente que el ensayo presentaba muchas ventajas. En referencia a los exámenes, los maestros utilizaban un cuestionario adaptado especialmente, que permitía revelar en la respuesta, no solo los conocimientos fundamentales de los programas, sino también la aptitud para interpretarlos, y esto era considerado un medio de comprobación superior al del examen habitual que se hacía, sobre una sola bolilla del programa.

En uno de los cuartos grados, agregaba, con motivo de una carta recibida de parte de los alumnos de la escuela Joaquín V. González de la ciudad de La Plata, donde solicitaban el envío de ejemplares de la flora regional, se realizó una interesante actividad que abarcó temas de las distintas asignaturas y terminó con el despacho de una encomienda conteniendo los ejemplares recolectados por los alumnos. Otro aspecto muy positivo de la experiencia, era que habían 
recibido la visita de delegaciones de profesores y alumnos de las escuelas de Esperanza, Rafaela y San Justo, interesados en conocer la forma en que se estaba aplicando la Escuela Nueva en la Normal de Paraná.

Más allá de estos logros obtenidos, Rodríguez mencionaba que una de las dificultades más serias que tenían en ese momento, era la que surgía de la actuación de los estudiantes del curso de magisterio - casi todas mujeresque hacían las prácticas docentes en esos grados, de una manera rutinaria y tradicional. Consideraba indispensable "despertar en el practicante" el interés por conocer verdaderamente la orientación general de este trabajo innovador, su función y la responsabilidad que debía tener en este tipo de clases. El problema era que no existía una vinculación real entre la cátedra de Pedagogía y la labor que se realizaba en la Escuela de Aplicación. En la actualidad, el practicante quedaba "perplejo y desorientado ante inevitables contradicciones entre la teoría y la práctica". El otro inconveniente, mencionaba, era la gran cantidad de practicantes que había para cada grado. Esto hacía que cada futuro maestro viera reducida la carga horaria de su práctica pedagógica, impidiéndole, además, disponer de más tiempo para comprender acabadamente los principios escolanovistas que se estaban aplicando.

En 1937, anunciaba la regente, se habían implementado los nuevos Programas de Asuntos en la Escuela de Aplicación, que no les habían presentado mayores dificultades a los docentes, porque resultaban una continuidad de la experiencia iniciada en 1931. Efectivamente, estos programas, pensados originalmente para las escuelas primarias de la ciudad de Buenos Aires, estaban en línea con lo que se venía haciendo en Paraná (CONSEJO NACIONAL DE EDUCACIÓN, 1937). Los principios escolanovistas se reconocían en el tipo de tareas propuestas para los niños: excursiones; construcciones de todo tipo en madera, cartón o papel; armado de colecciones y recortes; dibujo, modelado y canto; actividades que involucrasen el lenguaje hablado y escrito y la posibilidad de que expresaran emociones y sentimientos. ${ }^{13}$

Según decían los documentos orientadores, para trabajar por "asuntos", y al desarrollarlos sobre la base de las actividades de los educandos, no era posible pensar en un horario rígido. Si bien se asumía como una propuesta nacional, se podían identificar en ella elementos de Decroly y del Plan Dalton, entre otros. Se sugería, por ejemplo, para los primeros grados inferior y superior, el dictado diario de una hora para Matemáticas, una hora para Lectura-Escritura y dos

13 De acuerdo a algunos analistas, la Escuela Nueva promovió y alentó la expresión emocional y sentimental entre los maestros, profesores y alumnos. Ver, entre otros, Ascolani $(2010,2017)$ y Toro Blanco (2020). 
horas para "asunto". Para primer grado inferior, se proponían desarrollar los siguientes "asuntos": "Nuestra escuela" (mes de marzo) y "Mi hogar" (abril y primera quincena de mayo), por ejemplo. Durante tres días a la semana, debían dedicarse: media hora para Juegos y media hora para Ejercicios Físicos y los restantes días, media hora para Música. Respecto a los recursos didácticos necesarios, se recomendaba tener: biblioteca y museo del aula y de la escuela; terrario y acuario de la escuela; pajarera, gallinero, jaulas y jardín de la escuela; adornos; normas de higiene para el aula y la escuela; uso de tarjetas y otros elementos para el aprendizaje; y cuestionarios y tarjetas de trabajo para los grados superiores, a través de los cuales el maestro dirigía las actividades de los alumnos, ya fuese individualmente o en grupo, planteándoles problemas a resolver, indicándoles estudios a realizar y sugiriéndoles labores distintas para llegar al dominio del "asunto". De este modo, el docente señalaba el camino y el alumno lo recorría.

La regente recordaba que, desde el ensayo de Montoya en 1931, se había iniciado en la Escuela un proceso gradual de renovación de las formas de trabajo. Asimismo, el tema adoptado por los nuevos programas coincidía en algunos casos, con los "centros de interés" desarrollados anteriormente en la Escuela en primero, segundo y tercer grado. Además, la distribución horaria de los nuevos planes, mejoraba sustancialmente el trabajo a realizar. Sin embargo, en los grados superiores, la dificultad esencial que se les había presentado era la extensión de los Programas de Asuntos, en relación con el tiempo destinado a cada uno y las múltiples actividades que debían ejercitarse. Otra cuestión que resultaba no del todo adecuada para la implementación de los principios escolanovistas, decía la regente, era la gran cantidad de alumnos que había por aula en la primaria, y el número creciente de trabajos que cada docente debía corregir, hechos que impedían realizar un seguimiento individualizado e integral de cada estudiante. Así también, la falta de recursos suficientes para llevar a cabo con éxito estas experiencias, era constante y en buena medida debían ser cubiertas por los propios docentes y la Cooperadora. ${ }^{14}$

14 Los Programas de Asuntos estuvieron vigentes hasta la llegada del peronismo, que los sustituyó por los Programas de desenvolvimiento, que también adscribían a principios escolanovistas. En un informe del director de la Normal de Paraná correspondiente al año 1958, se mencionaba que se estaba implementando el Plan Dalton en los grados superiores (RODRÍGUEZ; PETITTI, 2021). 


\section{Consideraciones finales}

En este artículo analizamos las adaptaciones que hicieron dos profesoras de la Escuela Normal mixta de Paraná, de distintas ideas de pedagogos extranjeros asociados a la Escuela Nueva. Observamos que esta experiencia se destacó dentro del normalismo, por la cantidad de tiempo que duró en los primeros grados, más de seis años, -sin contar el ensayo que comenzó en el Jardín, en 1928- y terminó abarcando todos los grados de la Escuela de Aplicación -seis, algunos desdoblados-. En segundo lugar, mencionamos que ambas conocieron estas innovaciones a través de la prensa especializada, extranjera y nacional. Montoya además, tuvo la oportunidad de hacer un viaje a Europa, donde visitó a los representantes del escolanovismo y sus escuelas. Su ensayo alcanzó una difusión nacional e internacional, a partir del libro de Ferrière, que lo comentaba elogiosamente en sus páginas. Según explicaba, éste era una combinación de premisas postuladas por Decroly (los "centros de interés") y Montessori principalmente, de las cuales había elaborado una síntesis propia, que la llevó a confeccionar materiales diseñados por ella misma y adecuados al contexto paranaense. Rodríguez, por su parte, buscó continuar lo realizado por Montoya en los primeros grados de la primaria, e implementar el Plan Dalton, modelo originario de Estados Unidos, en los grados superiores. La regente decía haberlo conocido leyendo revistas como La Obra y comprando los materiales de la editorial Kapelusz.

Hemos visto que las dos manifestaban un conocimiento acabado de las experiencias originales, que les permitía identificar las dificultades que tuvieron para adaptar esas propuestas a la realidad local. Ambas indicaban qué aspectos habían podido implementar de los ensayos originales: lograron innovar en la organización del aula, instalando una mesa de arena, eliminando los bancos rígidos y agrupando a los niños alrededor de mesas y sillas que podían moverse fácilmente (Montoya); y transformar las aulas en Laboratorios y acondicionarlas para trabajar por grupos de asignaturas (Rodríguez). Consiguieron cierta flexibilización en los horarios para continuar con las actividades si los niños así lo deseaban, pudieron salir del edificio escolar y trasladar las experiencias de aprendizaje a la plaza y realizar varias excursiones (Montoya); promovieron el autogobierno, la autodisciplina y la libertad de movimiento de los estudiantes más grandes, así como la implementación de exámenes alternativos (Rodríguez); armaron bibliotecas en las aulas, y pudieron hacer uso de las piezas del museo y de las proyecciones (Rodríguez). 
Las máximas autoridades nacionales, acordaron en el año 1937, implementar los Programa de Asuntos que pretendían ser una versión nacional de la Escuela Nueva, donde se contemplaban varias de las actividades que se estaban haciendo en la Normal de Paraná: excursiones, trabajos manuales, dibujo, modelado, canto, y propuestas que promovieran la expresión libre de los niños. Desconocemos cómo se fueron desarrollando estos programas, pero en principio, tuvieron muy buena aceptación de parte de la regente.

En relación con las dificultades, estas propuestas innovadoras debieron ceñirse a los programas oficiales y a los horarios de entrada y salida (Montoya y Rodríguez), el número de aulas fue insuficiente para poder organizar los Laboratorios por cada asignatura (Rodríguez) y para establecer los talleres de plástica, madera y cartón (Montoya), la cantidad de matrícula generó que las aulas tuvieran mucho más alumnos de los deseables (Montoya y Rodríguez), faltó un parque o una huerta para poder realizar siembras y observaciones para las clases de Naturales (Montoya) y resultaron escasos los libros en las bibliotecas de aulas (Rodríguez). A estos inconvenientes, se sumó, según mencionaba Rodríguez, la incomprensión e indiferencia de padres y de estudiantes que se resistían a cambiar sus hábitos de estudio; y la escasa articulación que hubo con el curso de magisterio, a cuyos estudiantes les resultó muy difícil llevar a cabo las prácticas en esos grados de primaria.

En suma, a través del testimonio de estas dos profesoras, quedaba en evidencia la ambigüedad con la que actuaban los altos funcionarios del Ministerio nacional, que, si por un lado, autorizaban la implementación de innovaciones y decían querer modificar a la escuela tradicional, por el otro, parecían desconocer que dichas innovaciones requerían generar consensos amplios, una inversión alta de recursos, modificar la normativa y mejorar sustancialmente las condiciones edilicias y la disponibilidad de aulas, entre otras cuestiones. Esto hizo que finalmente, tanto en la Normal de Paraná como en otras instituciones, muchos de estos ensayos fuesen complejos de sostener en el tiempo, con la sola voluntad de los maestros, regentes y directores. 


\section{REFERENCIAS}

ASCOLANI, Adrián. Libros de lectura en la escuela primaria argentina: civilizando al niño urbano y urbanizando al niño campesino (1900-1946). Educação em Revista, v. 26, n. 1, p. 303-326, 2010.

ASCOLANI, Adrián. Concepciones reformistas en torno a las funciones de la educación primaria rural Argentina (1930-1960). Dialogia, São Paulo, n. 25, p. 43-68, 2017.

BRASLAVSKY, Berta La querella de los métodos en la enseñanza de la lectura: sus fundamentos psicológicos y la renovación actual. Buenos Aires: Unipe, [1962] 2014.

BOSCH, Beatriz. Irradiación del normalismo paranaense. Buenos Aires: Academia Nacional de Historia, 1993.

CALDO, Paula; FERNÁNDEZ, Sandra. Biografía, historia y mujeres: la revisión de un vínculo complejo a partir del caso de Olga Cossettini, 1898-1987. Avances del Cesor, n. 7, p. 115-139, 2010.

CARLI, Sandra. Modernidad, diversidad cultural y democracia en la historia educativa entrerriana (1883-1930). En: PUIGGRÓS, Adriana (dir.); OSSANA, Edgardo (coord.). La educación en las provincias y territorios nacionales (1885-1945). Buenos Aires: Galerna, 1993. p. 185-237.

CARLI, Sandra. Niñez, pedagogía y política. Transformaciones acerca de la infancia en la historia de la educación argentina entre 1880-1955. Buenos Aires: Miño y Dávila, 2002.

CARUSO, Marcelo. ¿Una nave sin puerto definitivo? Antecedentes, tendencias e interpretaciones alrededor del movimiento de la Escuela Nueva. En: PINEAU, Pablo; DUSSEL, Inés; CARUSO, Marcelo. La escuela como máquina de educar: tres escritos sobre un proyecto de la modernidad. Buenos Aires: Paidós, 2013. p. 93-134.

CONSEJO NACIONAL DE EDUCACIÓN. Programas para las escuelas comunes de la Capital Federal. Buenos Aires: Talleres Gráficos, 1937.

CUCUZZA, Héctor R. Desembarco de la Escuela Nueva en Buenos Aires: heterogéneas naves atracan en puertos heterogéneos. Revista Brasileira de Pesquisa, Salvador, n. 5, p. 310-329, 2017.

DE MIGUEL, Adriana et al. Traducción pedagógica, nacionalización canónica y contrato de lectura en la historia del canon escolar normalista. Ciencia, Docencia y Tecnología, Buenos Aires, v. 7, n. 7, p. 93-109, 2017.

DIP, Hiram. La Escuela Nueva en años conservadores. En: CARRIZO, Bernardo; GIMÉNEZ, Juan Cruz (coord.). La politica en las tramas educativas. Entre Ríos: La Hendija, 2017. p. 23-44.

FERRIÈRE, Adolphe. La escuela activa en América Latina. Madrid: Bruno del Amo, 1936. 
FIGUEROA, Sara. Escuela Normal de Paraná. Paraná: Predassi Impresores, 1934.

FILHO, Lorenzo. Introducción al estudio de la Escuela Nueva. Buenos Aires: Kapelusz, 1964.

FRECHTEL, Ignacio. La visita de Adolphe Ferrière a la Argentina en 1930: el movimiento de la escuela nueva y el flujo global de las ideas pedagógicas. Un caso para pensar las relaciones centro periferia, ponencia presentada en ISCHE 39, Buenos Aires: UMET, 2017.

FRECHTEL, Ignacio. La reforma de programas escolares de 1936 en Argentina: prensa pedagógica y Escuela Nueva. Tempos e Espaços em Educacão, São Cristovão, SE, v. 13, n. 32 , p. 1-18, 2020.

GIMÉNEZ, Juan Cruz. Politicas educativas y reformas pedagógicas en la Santa Fe de los años treinta. 2020. Tesis (Maestría en Ciencias Sociales) - Universidad Nacional del Litoral, Santa Fé, 2020.

REZZANO, Clotilde Guillén de. Hacia la Escuela Activa. Buenos Aires: Jesús Menéndez, 1934.

GVIRTZ, Silvina (comp.). Escuela nueva en Argentina y Brasil. Buenos Aires: Miño y Dávila, 1996.

GVIRTZ, Silvina. El problema de la escritura en la Escuela Nueva Argentina: de lo higiénico- pedagógico a lo psico-pedagógico en el discurso educativo. Revista da Faculdade de Educação, São Paulo, v. 24, n. 1, p. 1-17, 1998.

GVIRTZ, Silvina; BAROLO, Gabriela. A Escola nova na Argentina. Apontamentos locais de uma tradiçâo pedagógica transnacional. En: VIDAL, Diana Gonçalves; RABELO, Rafaela S. Movimento Internacional da Educação nova. Belo Horizonte: Fino Traço, 2020. p. 133-152.

IBARLUCÍA, Ricardo. Luis Juan Guerrero en la Facultad de Paraná: renovación filosófica y pedagogía moderna (1929-1930). Tópicos, Asociación Revista de Filosofía de Santa $F e$, n. 35, p. 36-68, 2018.

KUMMER, Virginia et al. "La Facultad de Ciencias Económicas y Educacionales". En: OSSANNA, Edgardo (dir.). Historia de la Facultad de Ciencias de la Educación de Paraná 1920- 1973. Paraná: Universidad Nacional de Entre Ríos, 2010, p. 75-118.

LEVORATTI, Alejo. La Educación Física y la gimnasia en la educación primaria y el nivel medio. Un estudio sobre las perspectivas teóricas en circulación (Argentina, 19581968). Revista de História e Historiografia da Educação, Curitiba, 2021. En prensa.

LUZURIAGA, Lorenzo. La educación nueva. Buenos Aires: Losada, [1943] 1958.

MONTOYA, Celia Ortiz A. de. Momentos culminantes en ciento cincuenta años de educación pública en Entre Ríos (1816-1966). Santa Fe: Colmegna, 1967. 
MONTOYA, Celia OrtizA. de. Nuestro ensayo de educación nueva. Boletín de Educación. Santa Fe, n. 89, p. 51-93, 1933.

NARODOWSKI, Mariano. Silencios y márgenes. La Escuela nueva en la historiografía educacional argentina. En: Gvirtz, Silvina (comp.). Escuela nueva en Argentina y Brasil. Buenos Aires: Miño y Dávila, 1996. p. 41-58.

OLANO, Ignacio. Los formatos escolares de las propuestas pedagógicas-didácticas del movimiento escolanovista argentino. Análisis de tres casos. 2020. Maestría (Ciencias Sociales con Orientación en Educación) - Facultad Latinoamericana de Ciencias Sociales, Buenos Aires, 2020.

OSSANNA, Edgardo et al. Historia de la Facultad de Ciencias de la Educación en Paraná (Argentina) (1920-1973). Ciencia, Docencia y Tecnología, Buenos Aires, n. 44, p. 73-110, 2012.

PADAWER, Ana. Las escuelas rurales del siglo XXI: las lecciones del maestro Luis F. Iglesias. Anales de la Educación común, Buenos Aires, n. 2, p. 25-32, 2017.

PELLEGRINI, Micaela; MOSSO, Agustina. Entre hermanas- entre mujeres: la vida cotidiana de Olga y Leticia Cossettini, Argentina, 1950-1980. Arenal. Revista de Historia de las mujeres, Granada, n. 24, p. 1-32, 2016.

PELLEGRINI, Micaela. Sensibilizar cuerpos para trabajar, sensibilizar cuerpos para consumir: la Escuela Nueva santafesina, Rosario, 1935-1950. Cartografias del Sur, Buenos Aires, n. 4, p. 136-159, 2016.

PÉREZ CAMPOS, Ana B. Escuela Normal de Paraná: construcciones discursivas de la nacionalidad argentina. Paraná. Santa Fe: UADER, 2016.

PUIGGRÓS, Adriana (dir.). Escuela, Democracia y Orden. Buenos Aires: Galerna, 1992.

RODRÍGUEZ, Laura Graciela; PETITTI, Mara. Historia de la Escuela Normal de Paraná (1871-1969). Buenos Aires: TeseoPress, 2021.

RODRÍGUEZ, María del Carmen. Memoria de la Escuela Normal. Caja 1934-1940. Archivo Escuela Normal de Paraná. Años: 1934, 1935, 1936, 1937.

ROITENBURD, Silvia. La Escuela Nueva en el campo adversario (1930-1943). En: GVIRTZ, Silvina (comp.). Escuela nueva en Argentina y Brasil. Buenos Aires: Miño y Dávila, 1996. p. 125-164.

ROMÁN, Mario S. Celia Ortiz de Montoya y las alternativas pedagógicas al Normalismo (1920-1932). Ciencia, Docencia y Tecnología, Buenos Aires, n. 16, p. 171-200, 1998.

ROMÁN, Mario S. La experiencia de Educación Integral Activa en la Escuela Normal de Paraná. En: ASCOLANI, Adrián. La educación argentina. Estudios de historia. Rosario: Arca, 1999. p. 197-208.

ROMÁN, Mario S. Viaje pedagógico, viaje intelectual: Celia Ortiz de Montoya: Tránsitos, trasiegos conflictos entre pedagogías. JORNADAS ARGENTINAS DE HISTORIA DE LA EDUCACIÓN, 14., 2006, La Plata. Anales [...]. La Plata: SAHE, 2006. 
ROMÁN, Mario S. "Para una historia de la cultura letrada en la Argentina. La enseñanza de la lectura y las prácticas de escritura en el ensayo de educación nueva (Paraná, Entre Ríos, 1931): un discurso alternativo al normalismo tradicional". Educación, Lenguaje y Sociedad, La Plata, n. 8, p. 55-73, 2011.

TEDESCO, Juan C. Educación y sociedad en la Argentina (1880-1945). Buenos Aires: Ediciones Solar, 1993.

TORO BLANCO, Pablo. Conselhos de viajantes: a Escola Nova e a transformaçâo do papel do profesor no Chile (1920-1930). Um olhar conciso da história transnacional e das emoções. En: VIDAL, Diana Gonçalves; RABELO, Rafaela S. Movimento Internacional da Educação nova. Belo Horizonte: Fino Traço, 2020. p. 153-174.

WELTI, Elisa; SERRA, María. La Escuela Nueva en Rosario: Olga Cossettini y la Escuela Serena Educadores con perspectiva transformadora. Santa Fe: FLACSO, 2018.

ZIPEROVICH, Rosa W. Memoria de una educadora: experiencias alternativas en la provincia de Santa Fe durante los últimos años de la década del 10, la del 20 y primeros años de 1930. En: PUIGGRÓS, Adriana (dir.). Escuela, Democracia y Orden. Buenos Aires: Galerna, 1992. p. 161-256.

Texto recibido en 21/06/2021.

Texto aprobado en 30/08/2021. 